\title{
Physico-chemical changes during fruit growth and developmental stages in yellow type passion fruit (Passiflora edulis f. flavicarpa Degener) accessions
}

\author{
Arkendu Ghosh ${ }^{1}$, Koyel Dey ${ }^{1 *}$, Fatik K. Bauri ${ }^{1}$ and A. N. Dey ${ }^{2}$ \\ ${ }^{1}$ Department of Fruits and Orchard Management, Faculty of Horticulture, Bidhan Chandra Krishi Viswavidyalaya, \\ Mohanpur, Nadia-741252 (West Bengal), INDIA \\ ${ }^{2}$ Department of Forestry, Faculty of Horticulture, Uttar Banga Krishi Viswavidyalaya, Pundibari, Cooch Behar- \\ 736165(West Bengal), INDIA \\ *Corresponding author. E-mail: koyelfruits@gmail.com
}

Received: September 7, 2016; Revised received: April 21, 2017; Accepted: September 18, 2017

\begin{abstract}
Physiological and biochemical changes during fruit growth, development and maturity of six yellow type passion fruit (Passiflora edulis f. flavicarpa Degener) accessions were studied at weekly interval after fruit set from 21 days to 91 days after fruit set and after dropping from vine also. Fruit growth of passion fruit followed a single sigmoid growth curve. Fruit length $(\mathrm{cm})$, diameter $(\mathrm{cm})$ and weight $(\mathrm{g})$ increased continuously $(25.24 \%, 33.13 \%$ and $75.08 \%$ respectively) (from the initial stage till maturity upto 84 days), which slightly declined at ripening stage. Fruits developed acceptable physico-chemical qualities with good colour, when harvested at 84 to 91 days after fruit set (DAF). The study further revealed that the days taken from fruit set to maturity and ripening, colour change, total soluble solids and acidity may be considered as the most reliable maturity indices for taking harvest decision in passion fruit.
\end{abstract}

Keywords: Fruit growth, Maturity, Passion fruit, Physico-chemical changes

\section{INTRODUCTION}

Passion fruit (Passiflora sp.) belongs to family Passifloraceae, of which purple ( $P$. edulis Sims) and yellow type ( $P$. edulis f. flavicarpa Degener) are common. It is a high valued crop having export potential due to its juicy flavour. In India, its cultivation is confined to Kerala, Tamil Nadu (Nilgiri hills and Kodai Kenal), Karnataka (Coorg) and northeastern states (Mizoram, Nagaland, Manipur and Sikkim) with an area and production of 9.11 thousand ha and 45.82 thousand tons (Joy 2010). This crop is gaining popularity in the north eastern hill states of India because of its adaptability, easy method of cultivation and higher yield (Average yield is $15 \mathrm{t} / \mathrm{ha}$ ) per unit area without much care. Due to its floral morphology, yellow passion fruit is an allogamous plant and self-in-compatibility of sporophytic type and cultivated for its edible fruits (Souza et al., 2006). Besides, it is also eaten as fresh by the people of this region due to its better eating quality. Fruits are utilized only after maturity, as immature fruits are highly acidic and contain less juice as well as flavour. Since, it is a climacteric fruit, ripening may also take place off the plant (Singh et al., 2006). It is known that the quality and storage life of fruits depend on various physico-biochemical changes, which occur during fruit growth, development and maturity. The fruits are generally harvested when they fall to the ground, leading to dehydration and microbial contamination, which ISSN : 0974-9411 (Print), 2231-5209 (Online) All Rights Reserved (C) Applied and Natural Science Foundation www.jans.ansfoundation.org reduces their shelf life and commercial value, resulting in economic prejudice (Meletti, 2011). Harvesting should be carried out when the fruits become ripe but still attached to the plant, allowing one to obtain them with better phytosanitary conditions, more uniform and with better quality, thus reducing losses and microbiological contamination (Amaro and Monteiro, 2001). The skin colour has been widely used as an indicator of the ideal harvesting point, since it was observed that fruits having $2 / 3$ yellow skin colour stage of ripeness which are very similar to one having $3 / 3$ yellow skin colour stage of ripeness (Janzantti and Monteiro, 2014). Therefore, it is important to recognize the ideal point to harvest the fruit. The information pertaining to the physico-chemical changes of yellow type passion fruit at different stages of growth, development and maturity is very scanty and for this, this work was carried out to study such changes in yellow type passion fruit accessions.

\section{MATERIALS AND METHODS}

Experimental site and design: The experiment was conducted at Research station of Mondouri, Bidhan Chandra Krishi Viswavidyalaya, Nadia, West Bengal with an elevation of $9.75 \mathrm{~m}$ above mean sea level $\left(23.5^{\circ} \mathrm{N}\right.$ latitude and $80^{\circ} \mathrm{E}$ longitude) during 20142015. Two years old bearing plants of six passion fruit accessions (Passiflora edulis f. flavicarpa), were se- 
lected for this study. The experiment was laid out in randomized block design with three replications and four vines were taken per replication (Fig. 1).

Collection of fruits: Few vines from all side of the plant were selected and then flowers were tagged with numbered metallic labels on the day of anthesis. The fruits initially set were marked in each vine. The first sample of the fruit was collected at 21 days after fruit set (DAF) and thereafter, samples were collected at weekly interval till maturity and dropping of fruits.

Observation recorded (Physico-chemical properties)

Fruit weight: Fruit weight was measured on the basis of 10 representative fruits with the help of electronic (digital) balance [Mettler Toledo PB153-L] and expressed in gram $(\mathrm{g})$.

Fruit length and breadth: Fruit length and breadth were measured from five randomly selected fruit during study with the help of digital vernier caliper and expressed in centimeters $(\mathrm{cm})$.

Rind thickness: Rind thickness of fruits was determined with the help of digital vernier caliper and expressed in millimeters ( $\mathrm{mm})$.

Juice content: The fruits from different accessions were weighed, cut and then juice was extracted from the pulp by squeezing and straining through muslin cloth under aseptic condition. The juice obtained was measured and the juice percent was determined from the volume of the juice divided by fruit weight and multiplied by 100 .

Total Soluble Solids (TSS ${ }^{\circ}$ Brix): Total soluble solids value is defined as the percentage concentration amount of sugar and soluble minerals present in any food or substances. TSS content of the fruit was recorded with the help of hand refractometer by the principle of total refraction which was calibrated at $0^{\circ} \mathrm{Brix}$ at $20^{\circ} \mathrm{C}$ with the help of a temperature correction cor-

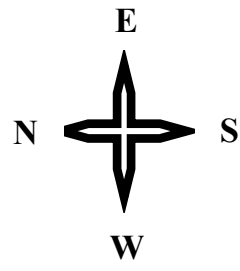

$\begin{array}{lll}\mathrm{A}_{1} \mathrm{R}_{1} & \mathrm{~A}_{3} \mathrm{R}_{2} & \mathrm{~A}_{4} \mathrm{R}_{3} \\ \mathrm{~A}_{2} \mathrm{R}_{1} & \mathrm{~A}_{5} \mathrm{R}_{2} & \mathrm{~A}_{6} \mathrm{R}_{3} \\ \mathrm{~A}_{6} \mathrm{R}_{1} & \mathrm{~A}_{1} \mathrm{R}_{2} & \mathrm{~A}_{5} \mathrm{R}_{3} \\ \mathrm{~A}_{3} \mathrm{R}_{1} & \mathrm{~A}_{6} \mathrm{R}_{2} & \mathrm{~A}_{2} \mathrm{R}_{3} \\ \mathrm{~A}_{4} \mathrm{R}_{1} & \mathrm{~A}_{2} \mathrm{R}_{2} & \mathrm{~A}_{1} \mathrm{R}_{3} \\ \mathrm{~A}_{5} \mathrm{R}_{1} & \mathrm{~A}_{4} \mathrm{R}_{2} & \mathrm{~A}_{3} \mathrm{R}_{3}\end{array}$

N.B. Here, $A$ and $R$ stands for Accession number and Repilcation number respectively

Fig. 1. Layout of experimental design. relation chart. A few drops of fruit juice from each sample were installed on the plate to record the refractometer reading in ${ }^{0}$ Brix (Mazumdar and Majumder 2003).

Titrable acidity content: The titrable acidity of the fruit juice was estimated by titrating against standard alkali solution $(0.1 \mathrm{~N} \mathrm{NaOH})$ using the phenolphthalein indicator and is expressed in percentage (Rangana 1977).

Total sugar and Reducing sugar content: The total sugar and reducing sugar content were estimated with the help of freshly made mixture containing equal volumes of Fehling's solution A \& B by copper reducing method (A.O.A.C 1984) using methylene blue as an indicator and was expressed in percentage (Mazumdar and Majumder 2003).

Ascorbic acid content: Ascorbic acid content of fruits was estimated based on the oxidation of ascorbic acid to Dehydro Ascorbic Acid and then to Diketo Gluconic acid followed by coupling with 2,4 DNPH and measured colorimetrically by UV/VIS spectrometer (Perkin Elmer, Lambda 25) expressed as mg per $100 \mathrm{~g}$ fruit pulp (Rangana 1977).

Fruit colour: The fruit colour was recorded with the help of Royal Horticulture Society Mini Colour Chart (fifth edition, 2007).

Statistical analysis: Analysis of variance (one way classified data) for each parameter was performed using op stat software (online version). The statistical analysis was done by following randomized block design (RBD) as per Gomez and Gomez (1983). The significance of different sources of variation was tested by error mean square by Fischer-Snedecor's ' $F$ ' test at probability level of 0.05 percent.

\section{RESULTS AND DISCUSSION}

Fruit weight: The details changes in fruit weight of different passion fruit accessions are presented in Table 1 . It has been shown that the fruit weight rapidly increased from 21 to 49 DAF (Days after fruit set) and thereafter the increase was slow till 84 DAF in almost all the genotypes. Rapid increase in fruit size and weight during initial stage could be due to fast cell differentiation and cell enlargement initially followed by slow rate (Patel et al., 2014). The maximum fruit weight was recorded in the accession-3 (102.16 g) followed by accession -2 (102.14 g), while the minimum fruit weight of $102.12 \mathrm{~g}$ was recorded in accession-4 at 84 DAF. Initially fruit growth was mainly associated with rind growth and later on seed and aril. The linear increase in fruit weight till 84 DAF and subsequent decline in weight towards ripening might be due to loss of water from fruits through transpiration.

Fruit length and breadth: Fruit growth (length and breadth) of passion fruit increased continuously from the initial stage of fruit development up to 84 days after fruit set (DAF) and thereafter, slightly decline till 
Arkendu Ghosh et al. / J. Appl. \& Nat. Sci. 9 (4): 2026 -2032 (2017)

Table 1. Changes in fruit weight ( $\mathrm{g}$ ) of passion fruit accessions (Passiflora edulis $\mathrm{f}$. flavicarpa Degener) at different stages of fruit maturity.

\begin{tabular}{|c|c|c|c|c|c|c|c|c|c|c|c|c|}
\hline \multirow[t]{2}{*}{ Accessions } & \multicolumn{12}{|c|}{ Days after fruit set } \\
\hline & 21 & 28 & 35 & 42 & 49 & 56 & 63 & 70 & 77 & 84 & 91 & Dropped \\
\hline Acc-1 & 58.28 & 65.16 & 70.08 & 94.81 & 95.01 & 95.41 & 95.79 & 96.01 & 100.15 & 102.13 & 100.12 & 99.70 \\
\hline Acc-2 & 58.31 & 65.18 & 70.09 & 94.84 & 95.06 & 95.42 & 95.80 & 96.02 & 100.16 & 102.14 & 100.13 & 99.73 \\
\hline Acc-3 & 58.35 & 65.25 & 70.11 & 94.87 & 95.08 & 95.45 & 95.82 & 96.07 & 100.18 & 102.16 & 100.20 & 99.80 \\
\hline Acc-4 & 58.26 & 65.13 & 70.08 & 94.81 & 94.99 & 95.39 & 95.78 & 95.99 & 100.15 & 102.12 & 100.07 & 99.69 \\
\hline Acc-5 & 58.33 & 65.25 & 70.10 & 94.84 & 95.06 & 95.43 & 95.80 & 96.06 & 100.17 & 102.15 & 100.18 & 99.79 \\
\hline Acc- 6 & 58.30 & 65.18 & 70.09 & 94.81 & 95.05 & 95.41 & 95.80 & 96.02 & 100.16 & 102.13 & 100.13 & 99.72 \\
\hline $\operatorname{SEm}( \pm)$ & 0.009 & 0.030 & 0.030 & 0.028 & 0.062 & 0.025 & 0.040 & 0.030 & 0.030 & 0.029 & 0.023 & 0.014 \\
\hline $\mathrm{CD}$ at $5 \%$ & 0.030 & NS & NS & NS & NS & 0.080 & 0.130 & NS & NS & 0.092 & 0.075 & 0.043 \\
\hline
\end{tabular}

Table 2. Changes in fruit length (mm) of passion fruit accessions (Passiflora edulis f. flavicarpa Degener) at different stages of fruit maturity.

\begin{tabular}{|c|c|c|c|c|c|c|c|c|c|c|c|c|}
\hline \multirow[t]{2}{*}{ Accessions } & \multicolumn{12}{|c|}{ Days after fruit set } \\
\hline & 21 & 28 & 35 & 42 & 49 & 56 & 63 & 70 & 77 & 84 & 91 & Dropped \\
\hline Acc-1 & 5.10 & 5.42 & 5.61 & 5.70 & 5.87 & 6.04 & 6.12 & 6.24 & 6.30 & 6.41 & 6.34 & 6.22 \\
\hline Acc-2 & 5.13 & 5.43 & 5.66 & 5.71 & 5.88 & 6.06 & 6.15 & 6.26 & 6.34 & 6.43 & 6.35 & 6.25 \\
\hline Acc-3 & 5.15 & 5.44 & 5.69 & 5.71 & 5.91 & 6.10 & 6.18 & 6.28 & 6.34 & 6.45 & 6.36 & 6.25 \\
\hline Acc-4 & 5.08 & 5.41 & 5.59 & 5.70 & 5.85 & 5.95 & 6.11 & 6.23 & 6.28 & 6.40 & 6.33 & 6.21 \\
\hline Acc-5 & 5.14 & 5.44 & 5.66 & 5.71 & 5.89 & 6.09 & 6.18 & 6.26 & 6.34 & 6.44 & 6.35 & 6.25 \\
\hline Acc- 6 & 5.10 & 5.42 & 5.65 & 5.70 & 5.87 & 6.06 & 6.15 & 6.25 & 6.30 & 6.43 & 6.35 & 6.24 \\
\hline $\operatorname{SEm}( \pm)$ & 0.028 & 0.029 & 0.018 & 0.020 & 0.018 & 0.698 & 0.015 & 0.016 & 0.013 & 0.015 & 0.016 & 0.022 \\
\hline $\mathrm{CD}$ at $5 \%$ & NS & NS & 0.058 & NS & NS & NS & 0.049 & NS & 0.041 & NS & NS & NS \\
\hline
\end{tabular}

Table 3. Changes in fruit diameter (mm) of passion fruit accessions (Passiflora edulis f. flavicarpa Degener) at different stages of fruit maturity.

\begin{tabular}{|c|c|c|c|c|c|c|c|c|c|c|c|c|}
\hline \multirow[t]{2}{*}{ Accessions } & \multicolumn{12}{|c|}{ Days after fruit set } \\
\hline & 21 & 28 & 35 & 42 & 49 & 56 & 63 & 70 & 77 & 84 & 91 & Dropped \\
\hline Acc-1 & 4.81 & 5.10 & 5.19 & 5.52 & 5.65 & 5.69 & 6.09 & 6.14 & 6.23 & 6.42 & 6.33 & 6.20 \\
\hline Acc-2 & 4.82 & 5.11 & 5.23 & 5.53 & 5.66 & 5.74 & 6.09 & 6.15 & 6.23 & 6.42 & 6.35 & 6.23 \\
\hline Acc-3 & 4.83 & 5.14 & 5.25 & 5.55 & 5.69 & 5.79 & 6.14 & 6.17 & 6.26 & 6.43 & 6.39 & 6.26 \\
\hline Acc-4 & 4.79 & 5.07 & 5.19 & 5.50 & 5.63 & 5.64 & 6.04 & 6.14 & 6.21 & 6.39 & 6.33 & 6.20 \\
\hline Acc-5 & 4.82 & 5.13 & 5.24 & 5.53 & 5.67 & 5.75 & 6.10 & 6.16 & 6.25 & 6.43 & 6.35 & 6.25 \\
\hline Acc- 6 & 4.81 & 5.11 & 5.22 & 5.53 & 5.66 & 5.74 & 6.09 & 6.14 & 6.23 & 6.42 & 6.34 & 6.22 \\
\hline $\operatorname{SEm}( \pm)$ & 0.019 & 0.018 & 0.018 & 0.022 & 0.027 & 0.016 & 0.021 & 0.020 & 0.021 & 0.018 & 0.022 & 0.027 \\
\hline $\mathrm{CD}$ at $5 \%$ & NS & NS & NS & NS & NS & 0.050 & NS & NS & NS & NS & NS & NS \\
\hline
\end{tabular}

Table 4. Changes in rind thickness $(\mathrm{mm})$ of passion fruit accessions (Passiflora edulis f. flavicarpa Degener) at different stages of fruit maturity.

\begin{tabular}{|c|c|c|c|c|c|c|c|c|c|c|c|c|}
\hline \multirow[t]{2}{*}{ Accessions } & \multicolumn{12}{|c|}{ Days after fruit set } \\
\hline & 21 & 28 & 35 & 42 & 49 & 56 & 63 & 70 & 77 & 84 & 91 & Dropped \\
\hline Acc-1 & 11.85 & 8.25 & 7.89 & 6.89 & 6.29 & 5.92 & 5.65 & 5.36 & 4.95 & 4.75 & 4.55 & 4.41 \\
\hline Acc-2 & 11.88 & 8.26 & 7.93 & 6.91 & 6.30 & 5.93 & 5.66 & 5.41 & 4.96 & 4.79 & 4.56 & 4.47 \\
\hline Acc-3 & 11.91 & 8.27 & 7.94 & 6.95 & 6.36 & 5.94 & 5.68 & 5.41 & 4.97 & 4.82 & 4.58 & 4.52 \\
\hline Acc- 4 & 11.85 & 8.24 & 7.87 & 6.88 & 6.25 & 5.90 & 5.63 & 5.35 & 4.93 & 4.73 & 4.55 & 4.38 \\
\hline Acc-5 & 11.89 & 8.26 & 7.94 & 6.94 & 6.33 & 5.93 & 5.68 & 5.41 & 4.97 & 4.81 & 4.57 & 4.49 \\
\hline Acc- 6 & 11.86 & 8.26 & 7.92 & 6.90 & 6.30 & 5.92 & 5.65 & 5.38 & 4.95 & 4.76 & 4.56 & 4.46 \\
\hline $\operatorname{SEm}( \pm)$ & 0.028 & 0.026 & 0.017 & 0.019 & 0.009 & 0.024 & 0.019 & 0.016 & 0.028 & 0.010 & 0.028 & 0.014 \\
\hline $\mathrm{CD}$ at $5 \%$ & NS & NS & NS & NS & 0.029 & NS & NS & 0.052 & NS & 0.031 & NS & 0.046 \\
\hline
\end{tabular}

Table 5. Changes in juice content (\%) of passion fruit accessions (Passiflora edulis f. flavicarpa Degener) at different stages of fruit maturity.

\begin{tabular}{|c|c|c|c|c|c|c|c|c|c|c|c|c|}
\hline \multirow[t]{2}{*}{ Accessions } & \multicolumn{12}{|c|}{ Days after fruit set } \\
\hline & 21 & 28 & 35 & 42 & 49 & 56 & 63 & 70 & 77 & 84 & 91 & $\overline{\text { Dropped }}$ \\
\hline Acc-1 & - & - & 22.75 & 32.49 & 33.13 & 33.65 & 33.99 & 35.11 & 37.22 & 37.30 & 34.30 & 33.91 \\
\hline Acc-2 & - & - & 22.75 & 32.53 & 33.14 & 33.66 & 34.05 & 35.13 & 37.25 & 37.32 & 34.32 & 33.93 \\
\hline Acc-3 & - & - & 22.76 & 32.56 & 33.16 & 33.69 & 34.09 & 35.17 & 37.26 & 37.34 & 34.34 & 33.95 \\
\hline Acc- 4 & - & - & 22.72 & 32.49 & 33.11 & 33.64 & 33.97 & 35.06 & 37.22 & 37.28 & 34.29 & 33.90 \\
\hline Acc-5 & - & - & 22.76 & 32.53 & 33.16 & 33.67 & 34.06 & 35.14 & 37.25 & 37.32 & 34.32 & 33.94 \\
\hline Acc- 6 & - & - & 22.75 & 32.51 & 33.14 & 33.66 & 34.00 & 35.12 & 37.24 & 37.32 & 34.31 & 33.92 \\
\hline $\operatorname{SEm}( \pm)$ & - & - & 0.028 & 0.018 & 0.025 & 0.021 & 0.061 & 0.024 & 0.025 & 0.015 & 0.018 & 0.017 \\
\hline $\mathrm{CD}$ at $5 \%$ & - & - & NS & 0.013 & NS & NS & NS & NS & NS & NS & NS & NS \\
\hline
\end{tabular}


Arkendu Ghosh et al. / J. Appl. \& Nat. Sci. 9 (4): 2026 -2032 (2017)

Table 6. Changes in TSS ('brix) of passion fruit accessions (Passiflora edulis f. flavicarpa Degener)at different stages of fruit maturity.

\begin{tabular}{|c|c|c|c|c|c|c|c|c|c|c|c|c|}
\hline \multirow[t]{2}{*}{ Accessions } & \multicolumn{12}{|c|}{ Days after fruit set } \\
\hline & 21 & 28 & 35 & 42 & 49 & 56 & 63 & 70 & 77 & 84 & 91 & Dropped \\
\hline Acc-1 & - & - & 9.32 & 10.13 & 11.24 & 11.98 & 12.82 & 13.98 & 15.09 & 16.17 & 16.42 & 16.20 \\
\hline Acc-2 & - & - & 9.32 & 10.13 & 11.25 & 11.99 & 12.84 & 13.99 & 15.13 & 16.22 & 16.45 & 16.23 \\
\hline Acc-3 & - & - & 9.34 & 10.14 & 11.32 & 12.04 & 12.85 & 14.00 & 15.15 & 16.24 & 16.48 & 16.25 \\
\hline Acc- 4 & - & - & 9.30 & 10.12 & 11.22 & 11.90 & 12.82 & 13.96 & 15.08 & 16.16 & 16.41 & 16.20 \\
\hline Acc-5 & - & - & 9.33 & 10.14 & 11.27 & 12.03 & 12.85 & 14.00 & 15.13 & 16.22 & 16.45 & 16.24 \\
\hline Acc- 6 & - & - & 9.32 & 10.13 & 11.24 & 11.99 & 12.84 & 13.99 & 15.11 & 16.20 & 16.43 & 16.21 \\
\hline $\operatorname{SEm}( \pm)$ & - & - & 0.018 & 0.020 & 0.018 & 0.041 & 0.018 & 0.021 & 0.022 & 0.025 & 0.026 & 0.026 \\
\hline $\mathrm{CD}$ at $5 \%$ & - & - & NS & NS & 0.058 & NS & NS & NS & NS & NS & NS & NS \\
\hline
\end{tabular}

Table 7. Changes in titrable acidity (\%) of passion fruit accessions (Passiflora edulis f. flavicarpa Degener) at different stages of fruit maturity.

\begin{tabular}{|c|c|c|c|c|c|c|c|c|c|c|c|c|}
\hline \multirow[t]{2}{*}{ Accessions } & \multicolumn{12}{|c|}{ Days after fruit set } \\
\hline & 21 & 28 & 35 & 42 & 49 & 56 & 63 & 70 & 77 & 84 & 91 & Dropped \\
\hline Acc-1 & - & - & 7.30 & 7.39 & 7.74 & 7.91 & 6.65 & 6.55 & 6.35 & 5.22 & 4.83 & 4.67 \\
\hline Acc-2 & - & - & 7.33 & 7.41 & 7.76 & 7.95 & 6.65 & 6.56 & 6.38 & 5.24 & 4.86 & 4.69 \\
\hline Acc-3 & - & - & 7.31 & 7.41 & 7.75 & 7.94 & 6.65 & 6.55 & 6.37 & 5.23 & 4.83 & 4.69 \\
\hline Acc- 4 & - & - & 7.25 & 7.35 & 7.72 & 7.90 & 6.65 & 6.52 & 6.34 & 5.21 & 4.83 & 4.66 \\
\hline Acc-5 & - & - & 7.33 & 7.41 & 7.76 & 7.95 & 6.67 & 6.57 & 6.40 & 5.24 & 4.87 & 4.69 \\
\hline Acc- 6 & - & - & 7.34 & 7.45 & 7.77 & 7.98 & 6.64 & 6.58 & 6.42 & 5.25 & 4.88 & 4.69 \\
\hline $\operatorname{SEm}( \pm)$ & - & - & 0.024 & 0.023 & 0.018 & 0.023 & 0.023 & 0.015 & 0.012 & 0.021 & 0.036 & 0.026 \\
\hline $\mathrm{CD}$ at $5 \%$ & - & - & NS & NS & NS & NS & NS & NS & 0.037 & NS & NS & NS \\
\hline
\end{tabular}

Table 8. Changes in total sugar (\%) of passion fruit accessions (Passiflora edulis f. flavicarpa Degener) at different stages of fruit maturity.

\begin{tabular}{|c|c|c|c|c|c|c|c|c|c|c|c|c|}
\hline \multirow[t]{2}{*}{ Accessions } & \multicolumn{12}{|c|}{ Days after fruit set } \\
\hline & 21 & 28 & 35 & 42 & 49 & 56 & 63 & 70 & 77 & 84 & 91 & Dropped \\
\hline Acc-1 & - & - & 2.00 & 2.18 & 2.47 & 8.11 & 8.71 & 10.11 & 10.55 & 12.59 & 13.38 & 13.35 \\
\hline Acc-2 & - & - & 2.00 & 2.21 & 2.48 & 8.16 & 8.73 & 10.16 & 10.59 & 12.61 & 13.39 & 13.37 \\
\hline Acc-3 & - & - & 2.08 & 2.22 & 2.50 & 8.19 & 8.78 & 10.18 & 10.59 & 12.62 & 13.42 & 13.39 \\
\hline Acc-4 & - & - & 1.98 & 2.15 & 2.42 & 8.10 & 8.69 & 10.11 & 10.55 & 12.53 & 13.35 & 13.31 \\
\hline Acc-5 & - & - & 2.08 & 2.21 & 2.49 & 8.17 & 8.73 & 10.17 & 10.59 & 12.62 & 13.39 & 13.38 \\
\hline Acc- 6 & - & - & 2.04 & 2.21 & 2.47 & 8.14 & 8.72 & 10.15 & 10.56 & 12.59 & 13.39 & 13.36 \\
\hline $\operatorname{SEm}( \pm)$ & - & - & 0.030 & 0.020 & 0.022 & 0.023 & 0.030 & 0.028 & 0.023 & 0.027 & 0.020 & 0.029 \\
\hline CD at $5 \%$ & - & - & NS & NS & NS & NS & NS & NS & NS & NS & NS & NS \\
\hline
\end{tabular}

Table 9. Changes in reducing sugar (\%) of passion fruit accessions at different stages of fruit maturity.

\begin{tabular}{|c|c|c|c|c|c|c|c|c|c|c|c|c|}
\hline \multirow{2}{*}{ Accessions } & \multicolumn{12}{|c|}{ Days after fruit set } \\
\hline & 21 & 28 & 35 & 42 & 49 & 56 & 63 & 70 & 77 & 84 & 91 & Dropped \\
\hline Acc-1 & - & - & 1.27 & 1.42 & 1.85 & 2.88 & 3.40 & 3.58 & 4.18 & 4.29 & 5.12 & 5.13 \\
\hline Acc-2 & - & - & 1.28 & 1.43 & 1.89 & 2.90 & 3.43 & 3.61 & 4.20 & 4.31 & 5.14 & 5.16 \\
\hline Acc-3 & - & - & 1.29 & 1.45 & 1.91 & 2.94 & 3.44 & 3.63 & 4.20 & 4.34 & 5.20 & 5.17 \\
\hline Acc-4 & - & - & 1.25 & 1.42 & 1.82 & 2.88 & 3.39 & 3.55 & 4.17 & 4.27 & 5.08 & 5.13 \\
\hline Acc- 5 & - & - & 1.28 & 1.44 & 1.91 & 2.93 & 3.44 & 3.62 & 4.20 & 4.32 & 5.20 & 5.17 \\
\hline Acc- 6 & - & - & 1.28 & 1.43 & 1.86 & 2.88 & 3.43 & 3.59 & 4.19 & 4.29 & 5.13 & 5.14 \\
\hline $\operatorname{SEm}( \pm)$ & - & - & 0.030 & 0.031 & 0.009 & 0.033 & 0.029 & 0.023 & 0.026 & 0.025 & 0.018 & 0.026 \\
\hline $\mathrm{CD}$ at $5 \%$ & - & - & NS & NS & 0.027 & NS & NS & NS & NS & NS & 0.057 & NS \\
\hline
\end{tabular}

Table 10. Changes in ascorbic acid (mg/100g pulp) of passion fruit accessions (Passiflora edulis f. flavicarpa Degener) at different stages of fruit maturity.

\begin{tabular}{|c|c|c|c|c|c|c|c|c|c|c|c|c|}
\hline \multirow[t]{2}{*}{ Accessions } & \multicolumn{12}{|c|}{ Days after fruit set } \\
\hline & 21 & 28 & 35 & 42 & 49 & 56 & 63 & 70 & 77 & 84 & 91 & Dropped \\
\hline Acc-1 & - & - & 55.92 & 57.69 & 51.33 & 37.58 & 37.69 & 34.58 & 34.49 & 25.51 & 22.59 & 21.56 \\
\hline Acc-2 & - & - & 55.93 & 57.66 & 51.36 & 37.62 & 37.71 & 34.59 & 34.51 & 25.52 & 22.62 & 21.58 \\
\hline Acc-3 & - & - & 55.94 & 57.65 & 51.32 & 37.65 & 37.73 & 34.62 & 34.53 & 25.53 & 22.65 & 21.61 \\
\hline Acc-4 & - & - & 55.92 & 57.69 & 51.31 & 37.56 & 37.67 & 34.57 & 34.47 & 25.48 & 22.58 & 21.56 \\
\hline Acc-5 & - & - & 55.91 & 57.74 & 51.33 & 37.63 & 37.71 & 34.60 & 34.51 & 25.52 & 22.63 & 21.59 \\
\hline Acc- 6 & - & - & 55.86 & 57.73 & 51.30 & 37.58 & 37.69 & 34.58 & 34.50 & 25.51 & 22.61 & 21.58 \\
\hline $\operatorname{SEm}( \pm)$ & - & - & 0.029 & 0.035 & 0.029 & 0.018 & 0.008 & 0.024 & 0.019 & 0.027 & 0.027 & 0.022 \\
\hline $\mathrm{CD}$ at $5 \%$ & - & - & NS & NS & NS & 0.055 & 0.027 & NS & NS & NS & NS & NS \\
\hline
\end{tabular}


Table 11. Changes in fruit colour of passion fruit accessions (Passiflora edulis f. flavicarpa Degener) at different stages of fruit maturity.

\begin{tabular}{|c|c|c|c|c|c|c|c|c|c|c|c|c|}
\hline \multirow[t]{2}{*}{ Accessions } & \multicolumn{12}{|c|}{ Days after fruit set } \\
\hline & 21 & 28 & 35 & 42 & 49 & 56 & 63 & 70 & 77 & 84 & 91 & Dropped \\
\hline Acc-1 & $\mathrm{G}$ & $\mathrm{DG}$ & $\overline{D G}$ & DG & $\mathrm{DG}$ & LG & $\mathrm{LG}$ & GY & GY & $\mathrm{LY}$ & $\mathrm{Y}$ & $\mathrm{Y}$ \\
\hline Acc-2 & G & DG & DG & DG & DG & LG & LG & GY & GY & LY & Y & Y \\
\hline Acc-3 & G & DG & DG & DG & DG & LG & LG & GY & GY & LY & Y & Y \\
\hline Acc-4 & G & DG & DG & DG & DG & LG & LG & GY & GY & LY & Y & Y \\
\hline Acc-5 & G & DG & DG & DG & DG & LG & LG & GY & GY & LY & Y & Y \\
\hline Acc- 6 & $\mathrm{G}$ & $\mathrm{DG}$ & $\mathrm{DG}$ & $\mathrm{DG}$ & $\mathrm{DG}$ & LG & LG & GY & $\mathrm{GY}$ & $\mathrm{LY}$ & $\mathrm{Y}$ & $\mathrm{Y}$ \\
\hline
\end{tabular}

$\mathrm{G}=$ Green, $\mathrm{DG}=$ Deep Green, $\mathrm{LG}=$ Light Green, $\mathrm{LY}=$ Light Yellow, Y=Yellow

Table 12. Maturity indices for yellow type passion fruit accessions (Passiflora edulis f. flavicarpa Degener).

\begin{tabular}{ll}
\hline Index & Passiflora edulis f. flavicarpa Degener \\
\hline Days from fruit set to harvesting (Days) & $84-91$ \\
Fruit colour & Light yellow to Yellow \\
TSS $\left({ }^{\circ}\right.$ Brix) & $>16$ \\
Acidity (\%) & $<4.8$ \\
\hline
\end{tabular}

fruit dropping in all accessions. Fruit growth of passion fruit followed a single sigmoid growth curve. The fruit reaches towards maturity showed the slow growth and declined at harvest maturity. The maximum fruit length and fruit diameter was recorded under accession -3 $(6.45 \mathrm{~mm}$ and $6.43 \mathrm{~mm})$, whereas minimum in accession-4 $(6.40 \mathrm{~mm}$ and $6.39 \mathrm{~mm})$ at $84 \mathrm{DAF}$. In case of dropped fruits, similar trend was also noticed (Table 2 and 3). Singh et al., (2006) reported that growth of passion fruit (Purple and Yellow type) showed the single sigmoid growth curve and slight decline in fruit size at 80 days after fruit set.

Rind thickness: As reported on Table 3, rind thickness of most of the accessions showed the decreasing trend from $21 \mathrm{DAF}$ to till ripening (84 DAF and dropped). The lowest (4.81 and $4.57 \mathrm{~mm}$ ) and highest (4.82 and $4.58 \mathrm{~mm}$ ) rind thickness was recorded in accession-5 and accession-3 respectively at 84 DAF and 91 DAF. The declining trend in rind content till maturity could be due to increase in seed size and water content into the rind during ripening of fruit. Similar results are also reported by Patel et al., (2014) in different passion fruit genotypes and Kishore et al., (2006) in purple colour passion fruits.

Juice content (\%): The juice formation started at 35 days after fruit set and increased rapidly up to $49 \mathrm{DAF}$ and continue comparatively slow rate of increase till $84 \mathrm{DAF}$ and then started decreasing at full ripe stage in most of the accessions. Findings are in accordance to Kishore et al., (2006) and Singh et al., (2006) in passion fruit. The maximum juice content was extracted in accession-3 (34.34 and 33.95\%) followed by accession -5 (34.32 and 33.94\%) and minimum in accession-4 (34.29 and $33.90 \%)$ at $91 \mathrm{DAF}$ and fruit dropped stage, respectively (Table 5). Loss of water from pulp and breaking down of polysaccharides tomono-saccharides during ripening might be the reason for reduction in juice content in passion fruit genotypes (Patel et al.,
2014).

Total Soluble Solids (TSS ${ }^{\circ}$ Brix): Data presented in Table 6 indicated that the TSS of fruit juice increased gradually with the advancement of maturity (till 91 DAF) and slightly decreased in the dropped fruits under all the accessions. A steady rise in TSS of the fruit was observed till final stage of harvesting. Similar results were also reported by Singh et al., (2006) in passion fruit. The increase in TSS content might be due to degradation of starch during later stage of harvest maturity as well as quick metabolic transformations in soluble compounds, mainly sugars. The TSS content in fruit was ranged from 16.41 to $16.48^{\circ} \mathrm{Brix}$ and the highest TSS content $\left(16.48^{\circ}\right.$ Brix $)$ was recorded from fruit harvested at $91 \mathrm{DAF}$ in accession-3 followed by accession-2 and 5 (16.45 Brix), accession-6 $\left(16.43^{\circ}\right.$ Brix $)$ and lowest $\left(16.41^{\circ}\right.$ Brix $)$ in accession-4.

Titrable acidity content: The fruit showed continuous and progressive increase in acidity up to $55 \mathrm{DAF}$ in most of the genotypes (Table 7) and afterwards, there was a continuous reduction in acidity till maturity (91 DAF and dropped fruits). The increase in acidity might be attributed to increased biosynthesis of organic acid during early stage of fruit growth. The decreased in acidity at later stages of fruit maturity was considered to be due to conversion of organic acids into sugars. Similar results were also reported by Deka et al., (2006) in Khasi mandarin and Kishore et al., (2006) in passion fruit. In this study, fruits at 91-day-old stage, lowest amount of acidity were recorded in accession-1, 3 and 4 (4.83).

Total sugar: After first observation to maturity (91 DAF), total sugars content showed a continuous increase (Table 8) in all the genotypes. Total sugars were increased rapidly at later stage of fruit development for all genotypes. The maximum total sugar content was recorded in accession-3 (13.42\%) followed by accession-2, 5 and $6(13.39 \%)$, while lowest $13.35 \%$ in 
accession-4 at 91 days after fruit set. The rapid increase in sugar content during later stage might be due to hydrolysis of starch into simple sugars and conversion of acids into sugars. The process of sweetening is accompanied by the conversion of starch into sugars. The increase in sugar might also be due to an increase in TSS and rise in glucose consumption by respiration (Sivaprasad et al., 2011). Similar trend was also reported by Kishore et al., (2006) in passion fruit and Deka et al., (2006) in pineapple.

Reducing sugar: The data presented in Table 9 showed an increasing trend in reducing sugar content of fruit juice till last harvesting. However, no significant difference was observed in reducing sugar due to accessions in most of the date of observations except 49 and 91 DAF. The highest reducing sugar content was analyzed in accession-3 (5.20\%) and lowest in accession-4 (5.08\%) at $91 \mathrm{DAF}$. The increase in reducing sugar might be due to hydrolysis of polysaccharides.

Ascorbic acid (mg per 100 g): The data presented in Table 10 revealed that the increasing trend in ascorbic acid content during early growth period (35-42 days) and thereafter it declined as fruit reaches towards maturity and ripening in all accessions. Higher ascorbic acid content during the initial fruit growth stage might be attributed to the adequate supply of hexose sugars in photosynthetic activity. The reduction in ascorbic acid content at later stages might be attributed to enzymatic oxidation of the L-ascorbic acid to dehydroascorbic acid during metabolic process (Neeraj et al., 2002). These findings were also in accordance with the findings of Dubey et al., (2003) in Khasi mandarin and Kishore et al., (2006) in passion fruit. Fruit harvested at 91 DAF produced the maximum ascorbic acid content of 22.65 and $22.63 \mathrm{mg} / 100 \mathrm{~g}$ for accession-3 and accession-5 respectively, while it was lowest in accession-4 (22.58 mg/100 g).

Fruit colour: Changes in fruit colour presented in Table 11 revealed that a gradual change in colour of passion fruit was observed during fruit growth and development. The rind colour of all the yellow type accessions showed the green colour up to $21 \mathrm{DAF}$ and thereafter changed into dark green colour till 49 DAF and then became light green, greenish yellow, light yellow and yellow colour at 56, 70, 84 and 91 DAF, respectively. These findings are in consonance with the findings of Kishore et al., (2006) and Singh et al., (2006) in passion fruit. Based on these finding, the some indices might be taken as the basic criteria for judging the maturity of passion fruit (Table 12).

\section{Conclusion}

The inevitable process of changes in physico-chemical composition turned out as a hallmark of maturity indices in yellow type passion fruit. Among several accessions, maximum fruit quality was attained during ma- turity in accession-3. It's fruit growth is followed a single sigmoid growth curve. For the first time, a set of developmental changes related physical and biochemical quality attributes have been identified for determining harvest maturity of this yellow type fruit in southern parts of West Bengal. Based on which this passion fruits may be harvest between 84 to 91 days after fruit set at light yellow to yellow stage with a TSS of $>16^{\circ}$ Brix. The composition and nutritive contents of this fruit are useful considerations when evaluating its uses and potential for value addition.

\section{REFERENCES}

Amaro, A.P. and Monteiro, M. (2001). Rendimento de extraç ao da polpa e características físico-químicas do maracujaamarelo (Passiflora edulis f. Flavicarpa Sims. Deg.) produzidopor cultivoorg^anico e convencional emrelaç aoacor da casca. Alimentos e Nutriç ao, 12: 171-84.

A. O. A. C 1984. In: Official methods of analysis, 14th edition, association of official agriculture

chemist, Washington, D.C. p.16.

Chandra, R. (1990). Biochemical changes during maturity and storage in guava fruits. Indian J. Hill Fmg., 8: 1621.

Deka, B.C., Sharma, S. and Borah, S.C. (2006). Post harvest management practices of mandarin.

Indian J. Hort., 63: 251-55.

Dubey, A.K., Patel, R.K. and Singh, A.K. (2003). Standardization of fruit maturity indices in Khasi mandarin (Citrus reticulata Blanco.) under Meghalaya. Ann. Agric. Res., 24: 559-62.

Gomez K. A. and Gomez A. A., (1983). Problem data. In: Statistical procedures for Agricultural

Research (2nd edition), Wiley-Inter science Publication (John Wiley and Sons); New York, USA. pp.275-315.

Joy, P.P. (2010). Status and prospects of passion fruit cultivation in Kerala. Pineapple Research Station (Kerala Agricultural University), Vazhakulam-686 670, Muvattupuzha, Ernakulam District, Kerala, India.

Janzantti, N.S. and Monteiro, M. (2014). Changes in the aroma of organic passion fruit (Passiflora edulis Sims f. flavicarpa Deg.) during ripeness. Food Sci. Tech., 59: 612-20.

Kishore, K., Bharali, R., Pathak, K.A. and Yadav, D.S. (2006). Studies on ripening changes in purple passion fruit (Passiflora edulis Sims). J. Fd. Sci. Tech., 43: 599602.

Mazumdar, B.C. and Majumder, K. (2003). Determination of chemical constituents. In: Methods of PhysicoChemical Analysis of Fruits, Daya Publishing house, Delhi. pp.93-139.

Meletti, L.M.M. (2011). Avançosnacultura do maracuja no Brasil. Revista Brasileira de Fruticultura, 33: 83-91.

Neeraj, M.S. and Bhatia, S.K. (2002). Effect of plastic packaging on biochemical parameters of

fruits during storage- A review. Haryana J. Hort. Sci., 31: 17.

Patel, R.K., Singh, A., Prakash, J., Nath, A. and Deka, B.C. (2014). Physico-biochemical changes during fruit growth, development and maturity in passion fruit genotypes. Indian J. Hort., 71(4): 486-493. 
Arkendu Ghosh et al. / J. Appl. \& Nat. Sci. 9 (4): 2026 -2032 (2017)

Rangana, S. (1977) Ascorbic acid. In: Manual Analysis of Fruit and Vegetable Products. Tata McGraw-Hill Publish. Comp. Ltd., New Delhi, pp. 94-101.

Singh, A., Patel, R.K., Babu, K.D. and Bhuyan, M. (2006). Flowering, fruiting and ripening physiology of passion fruit. Env. Ecol., 245: 693-97.

Sivaprasad, M., Policegoudra, R.S., Swaroop Kumar, H.M. and Aradhya, S.M. (2011). Changes in the biochemical and bioactive constituents of Indian hog plum (Spondias mangiferaWilld.) fruit during ambient storage. J. Hort. Sci. Biotechnol., 86 (3): 267-276.

Souza, M.M., Pereira, T.N.S., Dias, A.J.B., Ribeiro, B.F. and Viana, A.P. (2006). Structural, Hystochemical and Cyto - chemical Characteristics of the Stigma and Style in Passiflora edulis f. flavicarpa (Passifloraceae). Braz. arch. biol. technol., 49: 93-98. 